\title{
Le cinéma au temps de la guerre civile
}

Cinema in Civil War Times

Jacopo Rasmi

\section{OpenEdition}

Journals

Édition électronique

URL : http://journals.openedition.org/recherchestravaux/1102

DOI : 10.4000/recherchestravaux.1102

ISSN : 1969-6434

\section{Éditeur}

UGA Éditions/Université Grenoble Alpes

\section{Édition imprimée}

ISBN : 978-2-37747-065-5

ISSN : 0151-1874

\section{Référence électronique}

Jacopo Rasmi, «Le cinéma au temps de la guerre civile », Recherches \& Travaux [En ligne], 93 | 2018, mis en ligne le 26 octobre 2018, consulté le 08 septembre 2020. URL : http://journals.openedition.org/ recherchestravaux/1102; DOI : https://doi.org/10.4000/recherchestravaux.1102

Ce document a été généré automatiquement le 8 septembre 2020

(c) Recherches \& Travaux 


\title{
Le cinéma au temps de la guerre civile
}

\author{
Cinema in Civil War Times
}

Jacopo Rasmi

«Pourquoi ces vies, ne pas aller les écouter là où, d'elles mêmes, elles parlent? "1

\section{Anonymes}

1 Prenons le cinéaste québécois Rodrigue Jean pour commencer. Si son nom ne vous est pas familier, ne vous en préoccupez pas : cette lacune n'a rien d'exceptionnel. En plus d'une certaine ignorance concernant en général le cinéma francophone non français dans l'hexagone, son anonymat relève d'une stratégie personnelle très précise et très précoce. En saisissant son nom dans un moteur de recherche quelconque, la rareté des informations (directes) obtenues au sujet de sa personne et de son travail témoigne d'une stratégie de désidentification et de discrétion. Une image - celle d'un homme au regard mélancolique baissé, en noir et blanc, émergeant d'un fond de lumière flou - et une poignée d'entretiens (à l'écrit, sans trace audiovisuelle) représentent les seules références directes à Rodrigue Jean sur le web ${ }^{2}$. Rodrigue Jean ayant signé de nombreux longs-métrages connus et critiqués au Canada depuis plus de vingt ans, cette situation d'invisibilité ne peut qu'apparaître comme le signe flagrant d'une prise de position : un choix (éthique) de disparition, ou du moins d'abaissement radical du seuil de présence. Une décision qui instaure immédiatement une conversion entre des possibilités d'apparition d'autres vies peu visibles et la permanence de la sienne dans une zone de réserve. Cette disparition ou réduction de visibilité peut constituer le premier geste d'une politique d'anonymat et de coopération au sein de l'activité cinématographique dont nous tâcherons de retracer les indices et les raisons. Elle s'affirmera, en particulier, dans la région la plus documentaire du territoire balisé par ses travaux filmiques, celle de l'expérimentation d'Épopée - groupe d'action en cinéma. Cet enjeu de 
l'anonymat fait partie des réflexions qui animent le groupe, qui en parle en ces termes à l'occasion d'un entretien :

Dans le contexte d'Épopée, il y a une volonté de faire disparaitre l'auteur. Souvent, dans le milieu du cinéma, c'est vu comme une coquetterie, de la fausse modestie. Mais Épopée est réellement le produit de discussions, d'échanges constants, et de rapports qu'on pourrait qualifier d'énergétiques ${ }^{3}$.

2 Si le cinéaste place cette action cinématographique sous le signe d'une condition anonyme, c'est qu'elle est requise par le sens et la réussite du travail du collectif : taire des noms et cacher des images pour que d'autres apparences et d'autres voix puissent se manifester.

\section{Infâmes}

3 Bien que l'histoire d'Épopée appartienne à l'époque contemporaine (ayant commencé autour de 2010), il faut remonter le cours du temps pour en repérer les racines et se confronter ainsi à un délai d'achèvement aux enjeux politiques. Ce retard, on le verra, s'explique par la résistance d'un certain agencement hégémonique de l'univers audiovisuel ainsi que par le lent effort d'en élaborer un fonctionnement alternatif. Le suivi de cette expérience - inspirée par un séjour à Londres au début des années 1990 (pendant une bourse de résidence chorégraphique) - nous demande de reculer d'une vingtaine d'années. À cette époque, Rodrigue Jean a déjà commencé à associer son intérêt initial pour les arts performatifs à son attrait pour le travail cinématographique et il ne tardera pas à transformer l'invitation d'une amie à faire du bénévolat dans un centre londonien pour jeunes prostitués (Streetwise Youth) en une animation d'ateliers de vidéo avec ses usagers. Deux engagements, au moins, motivent son initiative : d'un côté, la possibilité offerte à ces personnes de mettre en récit et de recomposer des existences chaotiques, obscures et fragmentées; de l'autre, l'urgence de braver le tabou social imposé par les classes dominantes (leur morale et leurs moyens de communication) autour du phénomène de la prostitution masculine, souvent combiné à celui de la toxicomanie. Ce sont de véritables "vies infâmes", selon le terme que Foucault nous a légué. Leur infamie est le royaume des noms indignes où se forge leur redoutable anonymat: " criminel », " pédé ", " prostitué », " clochard », " toxico »... Malgré sa volonté de transformer ce travail en une création documentaire, Rodrigue Jean doit abandonner son projet à cause de l'absence d'infrastructures de production disponibles pour en permettre la réalisation. À cause de son refus de se soumettre à des ingérences très méticuleuses dues à la sensibilité socio-politique du sujet, le cinéaste devra renoncer aux moyens (logistiques, humains et financiers) proposés par la BBC et Channel 4 lors d'une première sollicitation. L'abandon des soutiens productifs entraine également l'abandon du projet filmique, dont la réalisation finalement sera reportée plutôt qu'oubliée.

4 L'occasion d'y revenir se présente à son retour au Québec, quelques dix ans plus tard, grâce à un encouragement de la part de l'ONF (Office national du film du Canada, dispositif de financement public de l'audiovisuel). En se penchant à nouveau sur l'expérience des travailleurs du sexe, Rodrigue Jean et son équipe trouvent un terrain de travail à Montréal dans le cadre du centre Action Sero Zero situé au cœur du quartier du Quadrilatère, au centre de la métropole québécoise. Pour le cinéaste, il s'agit de trouver un espace intermédiaire protégé où rencontrer ces sujets plutôt que d'aller les chercher et les filmer directement dans le territoire urbain où se dessine - 
entre les trafics de corps et de stupéfiants - leur extrême et frénétique vulnérabilité. Le film qui en résultera est donc le fruit d'une confiance établie d'abord avec les opérateurs d'Action Sero Zero (très prudents, notamment face au monde de l'information) et, ensuite, avec les jeunes qui fréquentent le centre. Le tournage est rythmé par plus d'une année de rendez-vous selon un principe de régularité qui défie la précarité et l'instabilité des sujets impliqués. Une telle durée semble pourtant nécessaire afin qu'ils puissent traduire leurs expériences en une narration orale et qu'ils trouvent un juste rapport au dispositif cinématographique. La garantie autant de leur parole que de leur assiduité - ce qui n'était pas du tout évident - a sans doute reposé sur le principe d'une relation hospitalière et attentive ainsi que d'un cadre simple et fixe d'entretiens frontaux, dans un lieu familier. Le plateau cinématographique se pense comme un refuge (bien qu'éphémère) pour des existences risquées et errantes qui obligent ainsi la troupe à fabriquer un dispositif de tournage adapté, à la hauteur de leur forme. Un film attentionné et patient de plus de deux heures où les témoignages des jeunes prostitués se relayent et évoluent dans le temps, sans qu'aucun commentaire ne se superpose à la vérité des corps et des paroles. Tel est le résultat final de cet atelier de travail : Hommes à louer (2008).

\section{Témoins}

5 Le film de Rodrigue Jean s'oppose au principe général qui régit, selon Foucault, la manifestation des existences infâmes :

Ce qui les arrache à la nuit où elles auraient pu, et peut être toujours dû, rester, c'est la rencontre avec le pouvoir: sans ce heurt, aucun mot sans doute ne serait plus là pour rappeler leur fugitif trajet. Le pouvoir qui a guetté ces vies, qui les a poursuivies, qui a porté, ne serait-ce qu'un instant, attention à leurs plaintes et à leur petit vacarme et qui les a marquées d'un coup de griffe, c'est lui qui a suscité les quelques mots qui nous en restent ${ }^{4}[\ldots]$.

6 En l'absence de ce travail cinématographique des protagonistes d'Homme à louer, nous n'aurions eu que les traces dérisoires et fatales de leur rencontre avec la machine du pouvoir. "Infâme » est la vie non ordinaire telle que les dispositifs gouvernementaux peuvent la décrire: par des comptes rendus policiers, par les fiches des assistants sociaux, par des documents médicaux, par des rapports juridiques... Ces documents n'auraient opposé à leur cavale silencieuse que les insipides classements (tels que " pauvre ", « malade ", « criminel », « toxicomane »...) dans lesquels est engloutie leur intense singularité. La fonction qui résiste à ce travail de surveillance et d'archivage du pouvoir serait selon Giorgio Agamben celle du « témoignage » :

Pour le distinguer de l'archive, qui désigne le système des relations entre le non-dit et le dit, appelons témoignage le système des relations entre le dedans et le dehors de la langue, entre le dicible et le non-dicible en toute langue - donc entre une puissance de dire et son existence, entre une possibilité et une impossibilité de dire. Penser une puissance en acte en tant que puissance, c'est-à-dire penser l'énonciation sur le plan de la langue, revient à inscrire dans la possibilité une césure qui distingue en elle une possibilité et une impossibilité, une puissance et une impuissance; et cela revient, dans cette césure, à situer un sujet ${ }^{5}$.

Alors que l'archive selon Foucault trace des rapports historiques et impersonnels de partage entre dit et non-dit (mais aussi vu et non-vu), le geste du témoin intervient comme une puissance qui fait appel à l'instance subjective : pour injecter du possible et de l'ingouvernable dans ces relations, en travaillant la matière d'un dehors irréductible 
aux catégories d'archivage. L'expérience filmique d'Hommes à louer s'inscrit sans doute dans ce dernier geste en fabriquant un espace de témoignage où l'ordre représentatif du pouvoir (enregistré par l'archive) est troublé par l'avènement des singularités (verbales, corporelles, affectives). Rodrigue Jean - comme beaucoup d'autres avant et après lui: Ferdinand Deligny, Pedro Costa, Wang Bing, Roberto Minervini, Pietro Marcello... - décide de ne pas se satisfaire des récits où le pouvoir piège la mémoire de ces vies pour aller faire ce que Foucault avait renoncé à faire. C'est-à-dire " aller les écouter là où, d'elles mêmes, elles parlent ", ces "existences réelles ", " obscures et infortunées », en dehors de « ce qu'on estime d'habitude digne d'être raconté ${ }^{6}$.»

\section{Impropres}

8 Afin d'achever Hommes à louer, une longue bataille a dû être engagée face aux injonctions des financeurs (autant du côté de la production que de celui de la distribution). Le soutien de l'ONF n'ayant pas été suffisant, Rodrigue Jean s'était adressé au monde de la production privée (InformAction) et télévisuelle par la forme du préachat (Radio-Canada). Ces liens avec le système marchand de financement et de diffusion du cinéma (avec la complicité de l'ONF) imposent très rapidement au développement $\mathrm{du}$ film les contraintes de l'exploitation commerciale. Il sera notamment question de réduire la durée de l'œuvre en coupant ses passages les plus dérangeants et non appropriés - selon des impératifs qui s'opposent à la justesse d'une certaine mesure et d'une certaine transparence respectées par le film face à ses protagonistes. L'équipe du film, épaulée par de nombreux membres de l'univers intellectuel et créatif québécois, s'oppose aux instructions péremptoires que l'infrastructure publico-privée dicte à la forme de l'œuvre. Cette dernière refuse au réalisateur de racheter les droits de son travail tout en évoquant les exigences commerciales («on est là pour vendre de la pub») afin de justifier sa violente intervention dans ses choix esthétiques. Dans la guerre froide déclarée par les revenus publicitaires et la capitalisation de l'investissement productif à l'attention sociale et à l'autonomie créative, l'avancement de la création atteint une impasse. En réaction à cette suspension, un appel est lancé contre l'« embargo inacceptable » qui interdit au travail de Rodrigue de circuler sous sa forme originale dévoilée lors de quelques projections exceptionnelles ${ }^{7}$. Finalement, le film pris en otage aura le droit d'être diffusé dans sa première version (140 minutes en $35 \mathrm{~mm}$ ) au prix du montage de deux autres versions conformes aux besoins du marché ( 52 minutes et 75 minutes) dont le cinéaste ne reconnaitra pas la paternité. Le constat est élémentaire et amer : il est extrêmement difficile de faire un autre cinéma (un cinéma d'écoute, d'écologie sociale) tout en demeurant à l'intérieur de l'infrastructure du cinéma commercial et de ses démarches d'appropriation. Pour créer d'autres images (contre les infamies), il faut créer autrement: il faut penser et pratiquer des méthodes, des économies et des organisations alternatives. Dans cette aventure s'affirme la nécessité d'un dispositif logistique, humain et financier à l'inverse d'un ordre commun impropre au travail de création qui engendrera le collectif Épopée. Ce collectif se fonde ainsi sur les principes d'une autonomie qui serait étrangère autant aux logiques (bureaucratiques et gouvernementales) de l'institution publique qu'aux intérêts (commerciaux) des agents privés. 


\section{Nues}

9 Les enjeux de la dispute - selon Rodrigue Jean - dépassent l'horizon purement cinématographique (celui d'une autonomie de création, par exemple) et s'inscrivent dans des frictions politiques plus profondes et cruciales. Ce conflit autour du visible, plus précisément, appartiendrait au mouvement d'une ample "guerre civile » qui informe tacitement nos communautés. Celle d'une protection de l'organisme social qui réprime ses membres marginaux en revendiquant qu' «il faut défendre la société8 ». L'interdiction d'une certaine visibilité ainsi que son contrôle dans le cadre du système audiovisuel de production et de diffusion nous renvoie donc à des phénomènes généraux de "gouvernementalité » des vivants que le cinéaste n'hésite pas à relier aux mécanismes biopolitiques étudiés par Foucault et Agamben :

Ce qui m'intéresse aussi dans le phénomeìne de la prostitution aujourd'hui, c'est la question de ce que Foucault appelle le biopouvoir, ou comment la société gère les corps. Voir comment on dispose à volonte philosophe italien qui a continué à travailler dans la foulée de Foucault, a développé un concept qu'il appelle Homo sacer. C'était cette catégorie d'hommes qui, dans la société romaine antique, pouvait être tuée sans qu'il y ait homicide au regard de la loi. Agamben montre comment, dans nos sociétés actuelles, la Déclaration universelle des droits de l'homme ne peut être applicable dans la mesure où existe toujours une semblable catégorie d'individus'.

Ce gouvernement des vies semble donc s'associer étroitement à celui de leurs formes : la puissance des apparences et des paroles des vivants fait l'objet de la même capture et gestion que leurs corps et de leurs conduites. Un certain gouvernement des conduites (ce qu'on peut faire ou pas) ainsi qu'un certain gouvernement de l'apparence (ce qu'on peu montrer et dire ou pas) présupposent la même condition de nudité de la vie, dépouillée de ses capacités immanentes. Cette vie nue, en tant que condition d'exercice de tout pouvoir, est celle qui est livrée à une gestion qui la transcende. Nos vies ne cessent pas de croiser les dispositifs gouvernementaux qui fonctionnent en présupposant leur nudité contre leur autonomie. Des dispositifs médicaux jusqu'aux opérations policières, les vivants sont systématiquement dépossédés de leur forme par la logique biopolitique du gouvernement. C'est en ce sens qu'on peut parler d'une " guerre civile » : à savoir d'un pouvoir qui s'exerce sur la communauté qui l'exprime et le constitue. Dans ce cadre, disposer des corps des autres (comme dans les politiques médicales ou policières) s'apparente à disposer de leurs images et de leurs voix. Cette violence semble ne devenir claire et intelligible que par ses aspects les plus radicaux : par exemple celui des abus policiers, celui des emprisonnements psychiatriques des individus anormaux ou encore celui des déportations des migrants. À ce propos, d'un regard provocateur mais lucide, Agamben nous a invité à reconnaitre dans la situation concentrationnaire le modèle inavouable des sociétés gouvernementales et de leurs mécanismes de réduction de la vie à la nudité, basés sur un état d'exception permanent. Le juif devient, chez le philosophe, la figure par excellence de cet homo sacer que Rodrigue Jean projette plutôt dans l'expérience des jeunes prostitués rencontrés à Londres et à Montréal. L'existence sociale des travailleurs du sexe toxicomanes d' Hommes à louer se réduit à une vie nue prise en charge par plusieurs dispositifs (sécuritaires) de gouvernement en tant que cas exceptionnel à administrer dans une défense sociale - qu'elle s'exprime par le gouvernement répressif des forces policières (thanatopolitique) ou par le gouvernement positif du système médicale (biopolitique). L'exclusion dont témoigne leur marginalisation ne représente qu'une phase du 
mécanisme qui en présuppose également l'inclusion (en tant qu'élément à discipliner et à gérer). Finalement, Homme à louer dresse un constat empirique de cette critique politique, grâce à une découverte émergée pendant une création où la réflexion théorique accompagne la pratique et les interrogations que s'y dévoilent.

Tout le travail va dans ce sens-là, dans la foulée de la réflexion sur le biopouvoir, dans la lignée de Foucault et d'Agamben. C'est l'histoire de l'Occident depuis la fin $\mathrm{du}$ Moyen Age, qui assiste à une mise en place de tous ces appareils qui, bien qu'ils se transforment, vont dans le même sens de l'asservissement du plus grand nombre. Dans Hommes à louer, c'est presque une thèse. La réflexion d'Agamben sur la vie nue, la vie sacrifiée, est entièrement là. Mais ce constat n'a pas précédé le film, c'est arrivé après coup, au moment du montage. Ça a été tellement long qu'il y a eu des lectures qui ont animé le montage. On en a profité. Ça a été une occasion extraordinaire de confirmer, de questionner certaines choses ${ }^{10}$.

11 Exposer ces vies plongées dans la prostitution et la toxicomanie signifie nous exposer, par ricochet, à notre propre condition de nudité qu'on a tendance à oublier lors de sa latence. C'est un rappel de la "guerre civile » que les dispositifs gouvernementaux ont toujours déjà déclarée, bien que d'une façon tacite et ambiguë, à leurs sociétés ${ }^{11}$. Dans cette fissure, selon un certain phénomène de contagion, le travail avec ces vies nues transforme le cinéma lui-même (et ses opérateurs, en particuliers) en une vie nue à soumettre à une économie supérieure de gouvernement.

\section{Sacrés}

Réalisé en temps de guerre civile, Hommes à louer est immédiatement absorbé dans le conflit. Cette situation d'exception menace de révoquer la légitimité de cette création. La «police» médiale s'avère ainsi une subtile variation de la police étatique. Cette équivalence, poussée à son extrême, finit par produire la superposition entre clandestinité visuelle et légale que la troupe d'Épopée expérimentera sur le tournage d' Insurgence (son film sur le Printemps érable ${ }^{12}$ ).

La violence a été subie comme chez n'importe quel autre manifestant. Souvent, on s'est mis dans des situations de vulnérabilité parce qu'on devait faire deux choses en même temps (tourner et éviter les assauts de la police). Plusieurs personnes ont été en choc traumatique, et on l'a été aussi ${ }^{13}$.

13 À travers leur alliance avec les soulèvements des étudiants, le cinéma et ses représentants forment une zone d'exception (ou de nudité, puisqu'ils sont dépouillés de leurs droits et de leur indépendance) contre et dans laquelle peut s'exercer violemment le gouvernement policier:

Certaines des personnes qui se sont retrouvées à Occupy sont aussi d'anciens travailleurs du sexe devenus sans-abris. Cette porosité des luttes ou des enjeux - entre l'itinérance, Occupy et le mouvement étudiant, est particulièrement intéressante. On se rend compte en étant près des travailleurs du sexe et des toxicomanes, qu'ils sont dans une situation de non-droit; ils vivent au quotidien dans un état policier. C'est un fait pour eux. Pendant le mouvement étudiant de l'hiver 2012, les Montréalais ont réalisé qu'eux aussi vivaient dans un état policier ${ }^{14}$.

Les événements du Printemps érable constituent un cas évident de réactivation de la guerre civile en tant que re-politisation d'une communauté inscrite dans la pure oikonomia de la biopolitique étatique ${ }^{15}$. Le profanation - au nom d'une autonomie, c'està-dire d'une forme-de-vie - de la souveraineté gouvernementale (comme dans le cas des révoltes étudiantes) entraîne le déclenchement immédiat de l'exception: 
soudainement déchu de son statut et de ses « droits », le citoyen fait la découverte de sa condition permanente de nudité vis-à-vis du pouvoir. Au cœur d'une Stasis comme celle du mouvement érable, l'étudiant comme le cinéaste font l'expérience d'une vulnérabilité et d'une exclusion (inclusive) de la gestion politique qui est ordinaire chez le toxicomane et le prostitué : ils se découvrent tous des « hommes sacrés»:

Ce dont on ne parle pas dans le projet, ni même dans les ateliers d'écriture, mais qui se communique par les conversations informelles, c'est que chacune des personnes avec qui on travaille est victime quotidiennement de violence de la part des institutions. Pour les toxicomanes et les travailleurs du sexe, l'habeas corpus n'a pas cours ${ }^{16}$.

15 Les situations quotidiennes des jeunes du Quadrilatère rejoignent ainsi celles de l'opérateur cinématographique ou de l'étudiant universitaire lors d'un mouvement contestataire dans l'espace d'une expérience commune de dépossession des corps et des gestes par une machine institutionnelle qui souhaite en disposer à leur place.

\section{Alliés}

16 Comme dans le contexte du Printemps érable et d'Occupy l'ouverture d'un front de conflit intérieur à la société rend immédiatement visibles les violences souterraines de l'ordre établi et dessine un geste de politisation des rapports qui n'étaient, avant, que subis d'une manière fatale ${ }^{17}$. Cette guerre civile devient aussi le foyer de nouvelles relations d'amitié et d'alliance à l'intérieur de la multitude sujette au monopole biopolitique de la guerre qui s'exerce dans nos économies gouvernementales. Dans le seul vrai commentaire du travail de Rodrigue Jean et d'Épopée paru en France métropolitaine, Marion Froger a voulu insister sur cette question (celle du partage d'une exclusion et d'une rupture qui s'accompagne de nouvelles occasions de solidarité et d'amitié) :

L'expérience physique et la vulnérabilité du corps politique dans l'affrontement avec les forces de l'ordre rejoignent celle du corps épuisé par la précarité et la marginalité, face à un même système de répression et de contrôle, dont chacun éprouve et conçoit, selon son expérience, la faillite démocratique et humaine ${ }^{18}$.

17 Ainsi le travail qu'Hommes à louer a inauguré avec les jeunes prostitués nous rappelle celui mené par Agamben autour des migrants apatrides marginalisés par les dispositifs des États nationaux ${ }^{19}$. Dans cette figure radicalement nue, le philosophe italien nous invite à observer autant l'emblème d'une expérience commune mais inavouable (nous tous, les homines sacri) que la condition d'une politique à venir où la forme d'une vie n'est plus appropriée et disposée par une machine gouvernementale. Ici, par un retournement stratégique, la forme des vivants ne serait plus octroyée et ôtée à l'envie par des dispositifs de souveraineté gouvernementale, ferait enfin l'objet d'un libre usage. Comme dans le travail au centre Action Sero Zero du groupe d'Épopée où l'exclusion partagée est renversée en une puissance de communauté qui fait évidemment écho à celui d'une longue série d'autres aventures cinématographiques qui se sont déroulées et se déroulent actuellement au sein des institutions où l'exception du pouvoir se pratique de la façon la plus ordinaire et évidente: dans les camps des migrants, dans les hôpitaux psychiatriques, dans les prisons... Par ces ateliers de réalisation organisés dans des espaces où la vie nue expérimente au quotidien l'exclusion inclusive, le cinéma (documentaire, en particulier) tente de re-politiser des vies et de leur restituer un véritable usage de leurs corps et de leur formes. Comme 
dans le cadre du collectif Épopée, il s'agit d'expériences qui invitent les sujets à se soustraire à la logique qui les réduit à une nudité gouvernable pour déployer leur autonomie de forme-de-vie.

Mais on avait passé beaucoup de temps avec les travailleurs du sexe et ces jeunes disaient : on a été l'objet de dispositifs toute notre vie, que ce soit l'orphelinat, la police, les travailleurs sociaux, la santé publique. On en a assez d'être documentés, on aimerait être des acteurs de nos vies. D'où l'idée de faire Épopée, un projet basé sur des ateliers d'écriture ${ }^{20}$.

La condition de possibilité fondamentale de ce travail est la mise en place d'une manière de faire du cinéma basée sur des rapports autonomes et coopératifs qui renvoie, plus amplement, à d'autres rapports sociaux et politiques possibles : à d'autres communautés.

\section{Brefs}

19 Confronté à la double émergence d'obstructions externes (celles de la production privée, avec la complicité de l'ONF) et de désirs internes (ceux des filmés de s'approprier la création filmique), le travail dans le Quadrilatère cherche une nouvelle forme adaptée aux obstacles et aux énergies qui se manifestent sur son terrain. Autour du nom anonyme d'Épopée, la nouvelle géométrie de la création filmique élabore un circuit cinématographique complètement autonome depuis la conception et la création jusqu'à la diffusion - sans recourir à aucune instance (privée ou publique) extérieure au groupe engagé dans la réalisation. Il faut accepter une réduction autogérée des moyens, sans renoncer pour autant à rien d'essentiel, grâce aux réseaux de solidarité ainsi qu'à la légèreté et à l'accessibilité des instruments numériques. C'est le constat du monteur Mathieu Bouchard-Malo :

C'est sûr que c'est dans la lignée d'Hommes à louer. C'est l'aboutissement d'une méthode de travail qui était là depuis longtemps, mais qui était un peu contrariée par des impératifs de production. On est à une époque où il y a un accès aux camé ras, accès aux salles de montage. Tout le monde peut prendre cette liberté-là. Mais les gens n'osent pas nécessairement proposer des choses sous une forme diffé rente ${ }^{21}$.

Un principe d'horizontalité et de coopération s'instaure à l'intérieur de cette troupe où - selon une logique où le respect des compétences spécifiques en présuppose aussi un partage au nom de l'égalité des intelligences - les activités se déroulent de la façon la plus collaborative et continue possible. Les singularités reliées par la fabrication d'un film sont multiples comme leurs apports : il y a des réalisateurs, des monteurs, des scénaristes, des opérateurs sociaux, des usagers du centre... Le travail d'Épopée représente la somme et la contamination de ces expériences différentes et de leurs capacités. L'indépendance technique et financière de la création ainsi que l'approche collective et empirique de la réalisation (sans aucun projet fixé en amont) engendrent une liberté formelle façonnée par les désirs émergents et les limites matérielles. Une telle recherche se déploie, d'ailleurs, sous le signe d'un choix de diffusion sur le web à travers une plateforme digitale qui ne veut pas évacuer une certaine rigueur cinématographique. Ayant renoncé à la salle au nom de l'autonomie, de la rapidité et de la souplesse de la distribution en ligne, la création d'Épopée se dirige vers des unités filmiques courtes, inscrites dans la veine du clip numérique (<http:// www.epopee.me>) : 
Pour nous, le Web est un moyen de diffusion. Les questions qu'on se pose sont avant tout des questions de cinéma, jamais des questions de plate-forme. Ce projet s'est construit au fur et à mesure, on n'avait pas une idée préconçue de la forme qu'il allait prendre. Une cohérence est ressortie de tous ces clips, même si on était dans l'inconnu à l'étape de la production. C'était à la fois un apprentissage du travail en groupe et une recherche de la forme appropriée pour chaque clip ${ }^{22}$. cohérence avec le support de diffusion et, en même temps, à l'économie du temps et des énergies des protagonistes dont l'engagement dans la création est limité aux brefs créneaux définis par les impératifs et l'instabilité de leur style de vie ${ }^{23}$. Même s'ils seront ensuite remontés dans des travaux plus complexes (de l'ordre du long métrage et de l'installation), l'immédiateté des clips d'Épopée représente la forme audiovisuelle la plus adéquate aux outils et aux participants de ce chantier cinématographique hétéroclite.

\section{Sollicitudinaires}

Deux veines se dessinent dans la série: d'abord des (micro) «fictions» - dont le scénario est tiré de l'expérience quotidienne de la vie dans la rue - qui donnent lieu à des plateaux plus improvisés que mis en scène. Ensuite, le format du «trajet » inventé par des désirs de cinéma moins écrits qui proposent à l'équipe d'Épopée et à sa caméra d'accompagner directement des moments et des mouvements quelconques d'une vie. Des rapports habituels (voire routiniers) entre des corps et des espaces urbains, des usages de la ville (c'est-à-dire des «territoires ${ }^{24} »$ ) tracent d'une manière cartographique le scénario de ces clips. Ils jouent leur vie, ils en sont protagonistes C'est ainsi que ces sujets vulnérables peuvent sortir de leur silencieuse disparition sociale ainsi que des fictions méfiantes et craintives que la société projette sur leurs vies. C'est ainsi qu'ils contre-fictionnent leur apparence « infâme » en l'émancipant des possibles sous-expositions et sur-expositions nuisibles dont ils font l'objet dans le système social et médiatique. Car nous n'existons pas seulement de notre existence de chair et de os, de nourriture et de santé - la seule existence que l'appareil biopolitique étatique semble en mesure de prendre en compte. Nous existons aussi d'une " existence sollicitudinaire » comme l'a affirmé Étienne Souriau :

[C]elle des êtres qui sont présents et existent pour nous d'une existence à base de désir, ou de souci, ou de crainte ou d'espérance, aussi bien que de fantaisie et de divertissement. [...] Leur caractère essentiel est toujours que la grandeur ou l'intensité de notre attention ou de notre souci est la base, le polygone de sustentation de leur monument, le pavois sur lequel nous les élevons; sans autre condition de réalité que cela. Complètement conditionnelles et subordonnées à cet égard, que de choses que nous croyons par ailleurs positives, substantielles, n'ont, quand on y regarde de près, qu'une existence sollicitudinaire ${ }^{25}$ !

Au fond, l'infamie (ciblée par le cinéma d'Épopée) n'est que la séparation d'un être de son apparence, n'est que la césure entre ce vivant et le foyer d'attention, de désir et d'imagination où s'établissent des liens sociaux plus justes et mutuels. Aux capacités de la création esthétique (autant ordinaire que professionnelle), comme à celle du cinéma, est confié le soin de l'existence sollicitudinaire de nous tous. 


\section{Citadins} sujets marginaux protagonistes des vidéos d'Épopée tentent de fuir les clichés qui les enveloppent dans une étreinte dangereuse. Ils secouent ainsi tout un système identitaire et ses cloisons, imposant au collectif autant qu'aux futurs spectateurs ce que Rodrigue Jean appelle « un travail de déclassement » : pour faire le cinéma d'Épopée (et le regarder, bien sûr) il faudrait d'abord "apprendre à se déclasser ${ }^{26}$ ». La création franchit ainsi cette distance entre nous les «normaux » et les laissés-pour-compte qui est sans cesse accentuée par nos vies citadines. Celle, par exemple, que le travail de Vincent Dieutre nous montrait dans son très touchant Jaurès (2012). Une fenêtre d'un immeuble respectable sur le canal Saint Martin à Paris séparait le cinéaste et sa caméra du campement de migrants situé en bas, à côté du cours d'eau. Il les observe (curieux, mélancolique, ému) du haut de son abri, comme nous observons tous les jours du haut de nos vies protégées celles précaires et délabrées des "pauvres » que nous croisons sans arrêt pendant nos trajets urbains. Sans jamais traverser la vitre, sans jamais atteindre une relation de contact et de contamination, Dieutre fantasme des correspondances intimes, des connexions silencieuses. Il projette par le récit de sa voixoff sa propre clandestinité infâme d'amant gay d'un homme à la pudeur raffinée (qui se consume à l'abri de l'appartement bourgeois) dans celle, légale, des migrants qui s'accrochent au bord du canal, au bord de l'attention des passants, au bord de son cadre. L'impuissance caressée par Jaurès est bravée par l'aventure d'Épopée qui confie la gestion de la création cinématographique (la forme de l'apparence et de la parole) à ces vies que Dieutre n'avait qu'observées et rêvées de loin. Ce n'est qu'en leur donnant la possibilité de (nous) jouer leur vie qu'il est possible de déjouer les séparations en mettant en place « une scène sociale virtuelle d'interaction permettant d'envisager, d'imaginer une autre communauté, plus ouverte, celle que nous fait éprouver cette expérience. » Autrement dit, comme l'affirme le même article consacré à l'aventure Épopée :

La valeur éthique de cette expérience cinématographique tient en premier lieu dans le rapprochement qu'elle permet entre Rodrigue Jean, les membres du collectif Épopée et ces hommes «vivant l'exclusion». Mais c'est aussi, du point de vue du spectateur, la ressource imaginaire que représentent pour lui les films qui en sont issus. Ressource imaginaire en ceci que les films lui permettent de parer à la rupture de socialité qu'il ressent, en tant que citadin, chaque fois qu'il traverse le centre-sud de Montréal et éprouve le malaise que suscite, dans la rencontre, l'épreuve de la distance qui le sépare des personnes en situation de précarité extrême ${ }^{27}$.

Rodrigue Jean était d'ailleurs convaincu que, même si «on est né dans une classe sociale ou dans un contexte ethno-culturel », « on n'est pas obligé d'y passer sa vie ${ }^{28}$ ». Qu'il est possible d'établir des nouvelles formes de "fraternité » par l'expérience cinématographique, celles d'une écologie différente des rapports qui agencent nos milieux urbains et de nos sociétés citadines ${ }^{29}$. De cette " fraternité » à venir les microépopées du Quadrilatère ne sont qu'une humble tentative : «quelques rudiments pour une légende des hommes obscurs ${ }^{30} »$. 


\section{NOTES}

1. Michel Foucault, "La vie des hommes infâmes ", dans Dits et écrits, Vol. II, Paris, Gallimard, 2001, p. 241.

2. Elle se trouve dans la fiche biographique du cinéaste compilée par Cinema politica: <https:// www.cinemapolitica.org/artists/rodrigue-jean>.

3. C'est l'affirmation du cinéaste dans "À la hauteur du sensible. Rencontre avec l'équipe d'Épopée » dans Spirale, hors-série 1, 2013, p. 33-40, p. 35.

4. Michel Foucault, « La vie des hommes infâmes », op. cit., p. 240-241.

5. Giorgio Agamben, « Auschwitz, l'archive et le témoin », dans Homo sacer. L'intégrale 1997-2015, Paris, Seuil, 2016, p. 913.

6. Michel Foucault, «La vie des hommes infâmes ", op. cit., p. 239-240.

7. On peut retrouver les traces de cette campagne dans les textes publiés par la revue québécoise Hors-champ, accessibles en ligne: voir notamment Sylvain L'Espérance, «Hommes à louer de Rodrigue Jean : un embargo inacceptable » et André Habib, « Tout n'est pas parfait ».

8. Voir notamment le cours de 1976 de Foucault au Collège de France : Il faut défendre la société, Paris, Seuil, 1997.

9. Rodrigue Jean et Matthieu Bouchard-Malo, «La poésie du vivant. Entretien avec André Habib », dans Hors-champ : <https://www.horschamp.qc.ca/spip.php?article333>.

10. Rodrigue Jean « Entretien avec Marie-Claude Loiselle », dans 24 Images, $n^{\circ} 136,2008$, p. 36-40, p. 39.

11. Ce terme, " guerre civile », est au cœur du titre d'un film récent de Rodrigue Jean (qui a suivi autant l'aventure d'Hommes à louer que celle d'Épopée) : L'amour au temps de la guerre civile (2014). Lors d'un entretien, il a revendiqué ce choix incompris par le journaliste en se référant clairement à la pensée d'Agamben: "Si on entend la guerre civile comme une lutte entre des formes-de-vies, votre incompréhension en est la démonstration ». (Rodrigue Jean, "L'amour au temps de la guerre civile: entretien avec le réalisateur", dans The Huffington Post: <https:// quebec.huffingtonpost.ca/2015/02/05/amour-temps-guerre-civile-realisateur-rodrigue-jeanentrevue_n_6622476.html?guccounter=1>)

12. L'expression «Printemps érable » désigne le plus important et radical mouvement de grève et de résistance des étudiants québécois qui a eu lieu entre le printemps et l'été 2012 contre les reformes néo-liberales du gouvernement, en particuliers celle de l'augmentation des frais de scolarité.

13. Rodrigue Jean dans "À la hauteur du sensible. Rencontre avec l'équipe d'Épopée ", op. cit., p. 35.

14. Rodrigue Jean dans "À la hauteur du sensible. Rencontre avec l'équipe d'Épopée ", op. cit., p. 37.

15. En continuité avec les références agambenienne qui ont balisé la réflexion précédente, nous nous appuyons à nouveau sur ses travaux pour ce qui concerne la notion de guerre civile (ou stasis) qu'il définit de la manière suivante : «La stasis ne provient pas de l'oikos, n'est pas une "guerre dans la famille», mais est une partie d'un dispositif qui fonctionne de manière semblable à l'état d'exception. De même que, dans l'état d'exception, la zoé, la vie naturelle, est incluse dans l'ordre juridico-politique de par son exclusion, de manière analogue, par le stasis, l' oikos est politisé et inclus dans la polis. » (Giorgio Agamben, Homo sacer. L'integrale 1997-2015, op. cit., p. 272)

16. Ibidem.

17. Il faut donc saisir la duplicité des mouvements incarnés par la logique de la stasis qui relève d'un jeu alterné entre politisation (de l'oikos vers la polis) et dépolitisation (de la polis vers l'oikos) ; 
«Nous devons donc concevoir la politique comme un champ de forces dont les extrêmes sont l' oikos et la polis : entre eux, la guerre civile marque le seuil par lequel l'impolitique se politise et le politique s'“économise". [...] Quand prévaut la tension vers l'oikos et que la cité semble vouloir se résoudre en une famille (certes d'une nature particulière), la guerre civile fonctionne alors comme le seuil où les rapports familiaux se repolisent; en revanche, quand c'est la tension vers la polis qui prévaut, et que le lien familial semble se relâcher, alors la stasis intervient pour recodifier en termes politiques les rapports familiaux. " (Giorgio Agamben, Homo sacer. L'integrale 1995-2015, op. cit., p. 272).

18. Marion Froger, «Épopée urbaine à Montréal », in Multitudes, $n^{\circ} 65,2016$, p. 190-197, p. 197.

19. Voir les analyses de son Moyens sans fins, Paris, Payot et Rivages, 2002.

20. Rodrigue Jean, « Entretien avec Marie-Claude Loiselle », op. cit., p. 36.

21. « À l'hauteur du sensible. Rencontre avec l'équipe d'Épopée », op. cit., p. 33.

22. Ibidem.

23. Il faut adapter la manière de travailler aux rythmes incertains et aux présences fuyantes des protagonistes de la création en réduisant la machine cinématographique à des éléments plus simples et rapides. C'est donc la forme de vie même des personnes impliquées qui demande un certain exercice d'immédiateté et une attention improvisatrice, comme le remarque Rodrigue Jean : "Le maximum de concentration et de présence qu'on peut obtenir des personnes qui consomment des drogues ne dure que quelques heures. Apprendre des textes, jouer, refaire des prises, est très difficile dans ce contexte. Surtout dans le cas des fictions, cette situation n'a pas permis de tourner beaucoup d'images. Lorsque quelque chose se manifeste, on n'est pas certain que ça puisse se reproduire." («À l'hauteur du sensible. Rencontre avec l'équipe d'Épopée ", op. cit., p. 35)

24. Nous pensons ici à la notion formulée par Deleuze et Guattari de «territoire » : « Le territoire n'est pas premier par rapport à la marque qualitative, c'est la marque qui fait le territoire. Les fonctions dans un territoire ne sont pas premières, elles supposent d'abord une expressivité qui fait territoire. C'est bien en ce sens que le territoire, et les fonctions qui s'y exercent, sont des produits de la territorialisation ». (Mille plateaux, Paris, Minuit, 1980, p. 388)

25. Étienne Souriau, Les différents modes d'existence, Paris, PUF, 1943, p. 79.

26. "À la hauteur du sensible. Rencontre avec l'équipe d'Épopée ", op. cit., p. 36.

27. Marion Froger, «Épopée urbaine à Montréal », op. cit., p. 191.

28. «À la hauteur du sensible. Rencontre avec l'équipe d'Épopée », op. cit., p. 36.

29. Rodrigue Jean évoque ce terme à propos des rapports instaurés pendant la création cinématographique : «Ultimement, toute la question de la fraternité c'est d'être renvoyé à soi, être en lien, établir des zones de continuité, entre la personne qui regarde et la personne qui est regardée, comme des couloirs de continuité. » (Rodrigue Jean et Matthieu Bouchard-Malo, «La poésie du vivant. Entretien avec André Habib », op. cit.)

30. Michel Foucault, « La vie des hommes infâmes », op. cit., p. 241.

\section{RÉSUMÉS}

L'article suit, dans son développement, le projet cinématographique autour des vies infâmes des travailleurs du sexe toxicomanes mené par le cinéaste québécois Rodrigue Jean, de la réalisation du film Hommes à louer (2008) jusqu'à l'expérience de cinéma collective, anonyme et autonome d' 
Épopée - groupe d'action en cinéma. Au cœur de cette expérience, le travail cinématographique est confronté aux violentes conditions d'une gouvernementalité biopolitique qui présuppose une "guerre civile» opérationnelle autant sur le plan physique et social que sur celui médial et perceptif. Le cinéma doit y prendre une position.

The text follows the development of a cinematic projet about the infamous lives of drug-addicted sex workers that leads the quebecois director Rodrigue Jean from the movie Hommes à louer (2008) to the collective, anonymous and autonomous experience of Épopée - groupe d'action en cinéma. In the middle of this experience, the cinematic work faces the violents conditions of a bio-politic gouvernamentality that implies a "civil war» (G. Agamben) active on the physical and social field as much as on the medial and perceptive one. In such a conflit, cinema shall take a stand.

\section{AUTEUR}

\section{JACOPO RASMI}

Jacopo Rasmi rédige actuellement une thèse autour de l'écologie des méthodes documentaires (au cinéma, mais aussi en littérature) au sein de l'Université Grenoble Alpes. Il est membre de la rédaction de La Revue Documentaires et de la revue Multitudes. Depuis quelques années, il programme à Grenoble le festival de cinéma documentaire Les rencontres autour du film ethnographique. 